\title{
Intensivierung
}

und ökonomische Reserven 

AUTORENKOLLEKTIV

\section{Intensivierung und ökonomische Reserven}

A KA DEMIE - VERLA G - BERLIN 1972 


\section{Russischer Originaltitel:}

Интенскфикация и резервы әкономики, Москва 1970

Verantwortlicher Redakteur:

Dr. sc. Krasovskij, V. P.

Ins Deutsche übersetzt von Dr. habil. Gerhard Krupp, Berlin

Wissenschaftlich bearbeitet durch Dr. habil. Alfred Tomm, Berlin

Erschienen im Akademie-Verlag GmbH Berlin, Leipziger Str. 3-4

Copyright 1972 by Akademie-Verlag - Berlin

Lektor: Dieter Graf

Lizenzmummer: $202 \cdot 100 / 208 / 72$

Umschlaggestaltung: Nina Striewski

Herstellung: IV/2/14 VEB Druckerei „Gotttried Wilhelm Leibniz 4 , 445 Gräfenhainichen/DDR · 3863

Bestellnummer: 6008 - ES 5 B 2

EDV-Nr.: 7521882

19,50 